\title{
Private Polizei und Bürgerinitiativen in Österreich und Deutschland
}

\section{Unterschiedliche Konzepte von "wir", "sie" und dem Staat in Europa}

\author{
Kerstin Stenius
}

$\mathrm{D}$ ie folgenden Beiträge von Irmgard Eisenbach-Stangl und Bettina Paul sind das Ergebnis einer Zusammenarbeit im Rahmen der International Society of Addiction Journal Editors (ISAJE). Die Herausgeber dreier Fachzeitschriften zur Alkohol- und Drogenforschung, der »Drugs: Education, Prevention and Policy « (Betsy Thom, England), der Wiener Zeitschrift für Suchtforschung (Irmgard Eisenbach-Stangl, Österreich) und der Nordisk alkohol- \& narkotikatidskrift (Kerstin Stenius, Finnland), haben sich zusammengetan, um Beiträge aus verschiedenen Ländern zu versammeln, die ein Thema behandeln, dass in verschiedenen Teilen Europas von Bedeutung ist: Die verschwimmenden Grenzen zwischen staatlicher und privater Kontrolle des öffentlichen Raums, des Drogenkonsums, der Kriminalität usw. Die Texte werden parallel in den Fachzeitschriften der verschiedenen Länder veröffentlicht, wobei sich die Neue Kriminalpolitik auf die deutschsprachigen Beiträge beschränkt. Darin geht es um unterschiedliche Beispiele für private Initiativen und Bürgerwehren, die sich als Reaktion auf eine allgemeine Kriminalitätsfurcht und insbesondere die Angst vor Drogenkonsumenten und »Fremden« verstehen.

Die Grazer Bürgerwehr, auf die sich Irmgard Eisenbach-Stangl bezieht, war eine Unternehmung der politischen Rechten. Dieser Versuch einiger Mitglieder der »Freiheitlichen Partei «, durch selbstorganisierte Patrouillen im öffentlichen Raum für Recht und Ordnung zu sorgen, rief vehemente und erfolgreiche öffentliche Proteste hervor, durch die die Bürgerwehr schon nach kurzer Zeit zur Aufgabe gezwungen wurde. Dennoch sieht Irmgard EisenbachStangl darin ein Anzeichen für einen verbreiteten Vertrauensverlust gegenüber der Polizei, sich den neuen Herausforderungen zunehmend globalisierter Probleme auf lokaler Ebene zu stellen. Xenophobie und Fremdenfeindlichkeit bilden den Nährboden für neue Organisationsformen auf Basis von Nachbarschaft, geteilten Problemen, gemeinsamer Lebensweise, Sprache oder eben auch »Rasse«. Die Verteidigung »unserer Lebensweise « ist demnach eher emotional als rational zu interpretieren, als Identitätsangebot in einer als chaotisch und unübersichtlich empfundenen Welt.

Die von Bettina Paul untersuchten Bürgerinitiativen in Hamburg beziehen sich auf diffuse, allgemeine Kriminalitätsängste. Sie bedienen die gleichen Feindbilder wie im österreichischen Beispiel und schüren die Angst vor Unbekannten und Fremden. Im Unterschied zu Österreich ist es jedoch gelungen, die privaten Proteste in politische Programme umzumünzen und statt selbstorganisierter Bürgerwehren das Ordnungsamt, die Polizei und die Sicherheitsdienste damit zu betrauen, unliebsame Personen aus dem öffentlichen Raum zu entfernen. So ist die Hamburger »Müllpolizei«, eine Einsatzgruppe des Ordnungsamtes, nicht nur für unangeleinte Hunde und weggeworfene Zigarettenkippen zuständig, sondern geht gezielt auch gegen Obdachlose, Betrunkene und Drogenkonsumenten im öffentlichen Raum vor. Dass marginalisierte Gruppen aus dem öffentlichen Raum verdrängt werden, verweist einerseits auf eine zunehmende Gleichgültigkeit gegenüber den sozialen Problemen. Andererseits gibt es gerade in den betroffenen Großstädten viel privates Engagement für diese marginalisierten Gruppen. Welche Form des Umgangs sich letztendlich durchsetzt, so resümiert Bettina Paul, hängt insbesondere davon ab, ob Vorurteile gegen Fremde und verschiedene Formen von Abweichung erfolgreich abgebaut werden können.

Das Easy Street project in Schweden (ausführlich behandelt in dem Aufsatz von Nilas Roth, den wir aus Platzgründen leider nicht mit ins Heft nehmen konnten) ist eine private Initiative, die Elemente von Gemeinwesenarbeit mit kommerziellen Interessen verbindet. Der Ausgangspunkt ist ähnlich wie bei den Beispielen aus Österreich und Deutschland: Soziale Proble- me. Easy Street will marginalisierte Jugendliche (re-)integrieren, indem gezielt ausländische, arbeitslose junge Menschen, die bereits straffällig geworden sind, z.B. durch Drogenkonsum, angeworben werden für Streifengänge in der UBahn, Hilfsdienste für Kriminalitätsopfer und bei diversen Kontrolltätigkeiten in Schulen und jugendlichen Freizeiteinrichtungen. Dieses Projekt hat sehr erfolgreich für staatliche Gelder und politische Unterstützung geworben. Aber es wurde auch kritisiert. Einige Mitarbeiter wurden des Drogenmissbrauchs überführt und weiterer Straftaten verdächtigt. Auch hat die Organisation nicht alle Leistungen erbracht, für die sie Mittel erhalten haben. Schließlich blieb unklar, welche Rechte und Pflichten die Mitarbeiter haben, wenn sie Zeugen von Straftaten werden oder wie ihre Funktion bezüglich des Schutzes der Privatsphäre einzustufen ist. Das Projekt bewegt sich in einer Grauzone zwischen öffentlichen und privaten Organisationsformen.

Alle drei Beispiele handeln davon, wie Bürger in Europa mit sozialen Problemen und Ängsten umgehen. Da es ausgewählte Beispiele sind, kann man daraus keine allgemeinen Schlussfolgerungen für ganz Europa ableiten, oder etwas über grundsätzliche Unterschiede zwischen den untersuchten Ländern sagen. Aber wir hoffen, dass diese Aufsätze weiterführende Diskussionen und Untersuchungen über das »wir «, »sie « und den Staat in verschiedenen europäischen Ländern anstoßen.

Wir möchten auch ankündigen, dass wir diese Form von grenzübergreifendem, mehrsprachigem Gedankenaustausch in Europa in unseren Zeitschriften fortsetzen werden.

Kerstin Stenius ist Vorsitzende der International Society of Addiction Journal Editors und Chefredakteurin der Nordic Studies on Alcohol and Drugs 